\title{
Dimethyl Fumarate
}

National Cancer Institute

\section{Source}

National Cancer Institute. Dimethyl Fumarate. NCI Thesaurus. Code C63670.

An orally bioavailable methyl ester of fumaric acid and activator of nuclear factor erythroid 2 [NF-E2]-related factor 2 ( $\mathrm{Nrf2}, \mathrm{Nfe} 2 \mathrm{l} 2$ ), with potential neuroprotective, immunomodulating and radiosensitizing activities. Although the exact mechanism of action throug h which dimethyl fumarate exerts its neuroprotective and immunomodulatory effects have yet to be fully understood, upon oral administration, dimethyl fumarate is converted into its active metabolite monomethyl fumarate (MMF) and MMF binds to Nrf2. Subsequently, Nrf2 translocates to the nucleus and binds to the antioxidant response element (ARE). This induces the expression of a number of cytoprotective genes, including $\mathrm{NAD}(\mathrm{P}) \mathrm{H}$ quinone oxidoreductase 1 (NQO1), sulfiredoxin 1 (Srxn1), heme oxygenase-1 (HO1, HMOX1), superoxide dismutase 1 (SOD1), gammaglutamylcysteine synthetase (gamma-GCS), thioredoxin reductase-1 (TXNRD1), glutathione S-transferase (GST), glutamate-cysteine ligase catalytic subunit (Gclc) and glutamate-cysteine ligase regulatory subunit (Gclm); this also increases the synthesis of the antioxidant glutathione (GSH). The intraneuronal synthesis of GSH may protect neuronal cells from damage due to oxidative stress. Dimethyl fumarate also appears to inhibit the nuclear factor-kappa B (NF-kB)-mediated pathway, modulates the production of certain cytokines and induces apoptosis in certain T-cell subsets. Its radiosensitizing activity is due to this agent's ability to bind to and sequester intracellular GSH, thereby depleting intracellular GSH and preventing its anti-oxidative effects. This enhances the cytotoxicity of ionizing radiation in hypoxic cancer cells. Nrf2, a leucine zipper transcription factor, plays a key role in redox homeostasis and cytoprotection against oxidative stress. 